\title{
SHORT COMMUNICATION Adequate Use of the Cottey Model for the Description of Conduction in Polycrystalline Films
}

\author{
C. R. TELLIER and A. J. TOSSER \\ Universite de Nancy I, Laboratoire d'Electronique, C.O. 140-54037 Nancy Cedex - France
}

(Received January 1, 1979)

An attempt has been recently made ${ }^{1}$ to give a new model of electrical conduction in polycrystalline metal films. Some points are discussed in this paper. In the theoretical calculations related to a new grain boundary scattering model for conduction in metals [1], the current density $J$ is written as

$$
J=e \int v_{x} \cdot f_{1}(\theta, \phi) d^{3} p
$$

where $e$ is the electron charge, $v_{x}$ the component of electron velocity $v$ in the direction of the applied electric field and $f_{1}$ stands for the deviation from the equilibrium function of Fermi distribution.

Eq. (1) has been given by $\operatorname{COTTEY}^{2}$ (p. 301) and is valid in spherical polar coordinates $(r, \theta, \phi)$ with:

$$
\begin{aligned}
& z=r \cos \theta \\
& x=r \sin \theta \cos \phi
\end{aligned}
$$

as shown in Figure 1.

Let us remember that in the Mayadas-Shatzkes conduction model $^{3}$ the grain boundaries are represented by two types of randomly spaced planes,

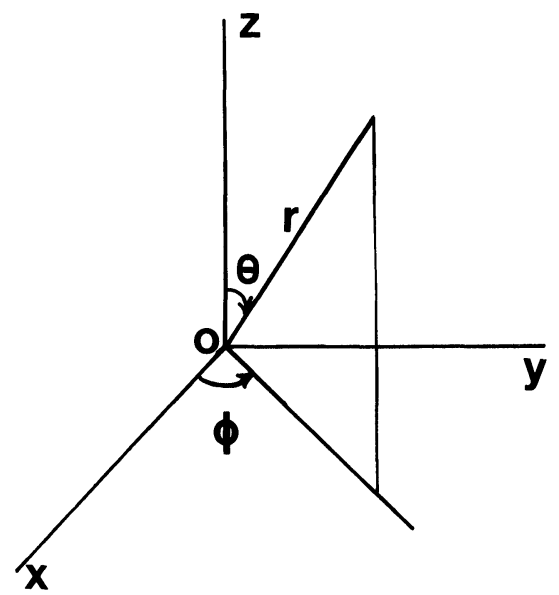

FIGURE 1 Definition of spherical polar coordinates. those parallel to the electric field, which produce only specular reflection, and those perpendicular whose effects are represented by a grain-boundary relaxation time $\left[2 F\left(\left|k_{x}\right|\right)\right]^{-1}$ (Refer to Mayadas and Shatzkes ${ }^{3}$, Eqs. $6 \mathrm{~b}$ and $7 \mathrm{~b}$ ) introduced in the Boltzmann equation.

The positions of the planes are defined by a Gaussian law [Mayadas and Shatzkes ${ }^{3}$ Eq. 3] whose standard deviation is $s$. In the limit $s \rightarrow 0$ no grain boundary resistivity is observed. This means that a periodic array of planes provides no resistance. However the average separation of the planes (which is identified with the average grain diameter $D$ ) could be used if the travelling distance of electrons $l$ satisfies the condition

$$
l \gg D
$$

The transmission of electrons across grain boundaries can be conveniently ${ }^{1}$ described with the COTTEY model, using the same assumptions as COTTEY $^{2}$, specially if the effect of boundaries on electrons can be described by an exponential function $P$ of the travelling distance. This feature requires that the effect of any boundary could be expressed by a given differential variation of $\boldsymbol{P}$ and that the electron path between two boundaries could be considered as a differential variation of $l$. In the new proposed model the first condition must be written in the form:

$$
r \approx 1
$$

Furthermore, under this assumption, the term $\vec{v} \cdot \overrightarrow{\operatorname{grad}_{r}} f$ can be neglected in the Boltzmann equation [Sondheimer ${ }^{4}$, Eq. 5].

The second condition must be expressed as:

$$
l \gg D^{\prime} \quad \text { for } \theta \approx \frac{\pi}{2}
$$

where $D^{\prime}$ is the boundary spacing measured in the vertical plane $(z-0-\rho)$ containing $\vec{v}$ (Figure 2 ).

If Eq. 4 is satisfied, the probability of electrons 


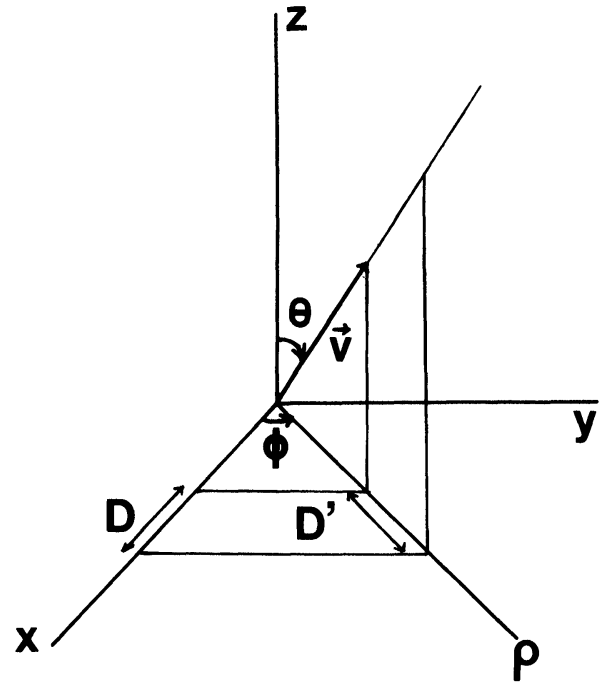

FIGURE 2 The geometry of the grain-boundary model.

travelling through $n$ grain boundaries $P$ is defined by $^{1,2}$

$$
\begin{aligned}
& P=r^{n} \\
& d P=-\frac{P}{\lambda_{D}} d l \quad \text { which gives } \\
& P=\exp \left(-1 / \lambda_{D}\right)
\end{aligned}
$$

where $\lambda_{D}$ is the mean free path associated with the influence of grain boundaries on the conduction electrons.

The travelling distance $l$ is calculated in the $(z-0-\rho)$ plane (Figure 3).

$$
l=\frac{n D^{\prime}}{|\sin \theta|}
$$

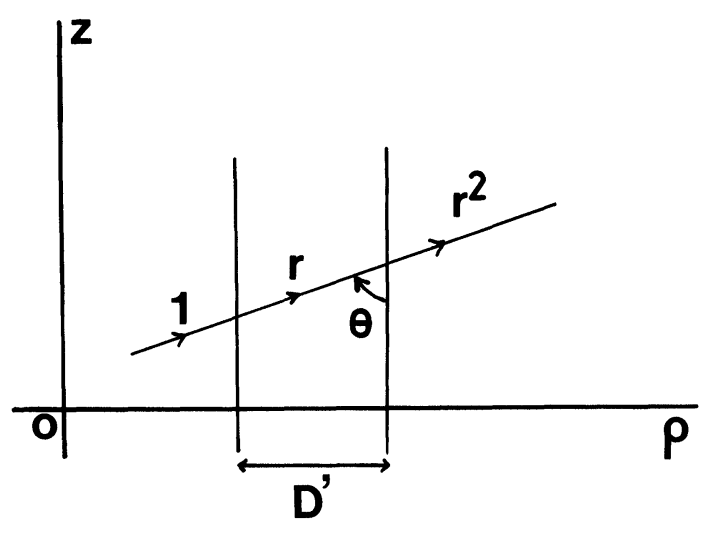

FIGURE 3 The geometry of the Warkusz model ${ }^{1}$. where (Figure 2)

$$
D^{\prime}=D /|\cos \phi| \text {. }
$$

Hence the theoretical expression of $\lambda_{D}$

$$
\lambda_{D}=\frac{D}{|\sin \theta||\cos \phi| \ln \frac{1}{r}}
$$

This expression does not agree with the expression previously proposed [Warkusz ${ }^{1}$, Eq. 4], since the author did calculate the travelling distance from the relation:

$$
l=\frac{n D}{|\sin \theta|}
$$

which is only valid for cylindrical grain boundaries. Hence, all equations derived are questionable [Warkusz ${ }^{1}$, Eq. 8, 9, 10, 11, 12].

Assuming that grain boundary and background scattering are independent, the mean free path $\lambda(\theta, \phi, r)$ for both scatterings is ${ }^{4,1}$.

$$
\frac{1}{\lambda(\theta, \phi, r)}=\frac{1}{\lambda_{0}}+\frac{1}{\lambda_{D}}
$$

where $\lambda_{0}$ is the bulk mean free path.

Solving the Boltzmann equation gives:

$\sigma_{g} / \sigma_{0}=\frac{3}{4 \pi} \int_{0}^{\pi} \int_{0}^{2 \pi} \frac{\sin ^{3} \theta \cos ^{2} \phi}{1+\frac{\lambda_{0}}{D}|\cos \phi||\sin \theta| \ln \frac{1}{r}} d \theta d \phi$

where $\sigma_{\mathrm{g}}$ is the grain boundary conductivity, i.e. the conductivity of an infinitely thick polycrystalline film, and $\sigma_{0}$ the bulk conductivity.

Eq. 12 is not easily integrated in these coordinates but more convenient coordinates could be used.

As it has been shown above Eq. 12 is valid if $r$ takes values near 1 .

Finally it appears that the new model recently proposed $^{1}$ is of slight physical interest and somewhat questionable whereas the alternative expression for Mayadas-Shatzkes equation is somewhat complicated. Further studies would be necessary.

\section{REFERENCES}

1. F. Warkusz, Electrocomp. Sc. Technol., 5, 197 (1978).

2. A. A. Cottey, Thin Solid Films, 1, 297 (1967-68).

3. A. F. Mayadas and M. Shatzkes, Phys. Rev. B, 1, 1382 (1970).

4. E. H. Sondheimer, Adv. Phys., 1, 1 (1952). 

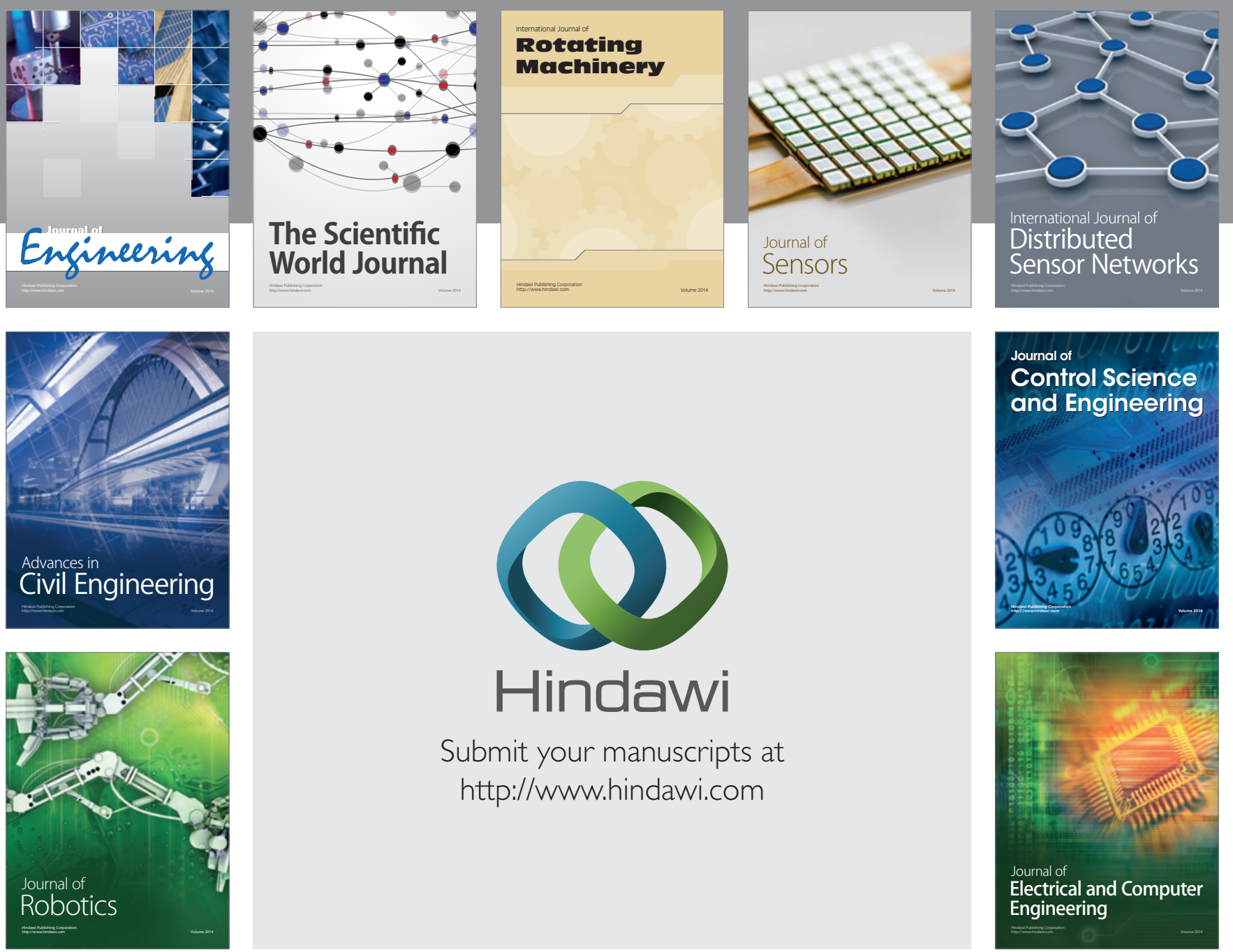

Submit your manuscripts at

http://www.hindawi.com
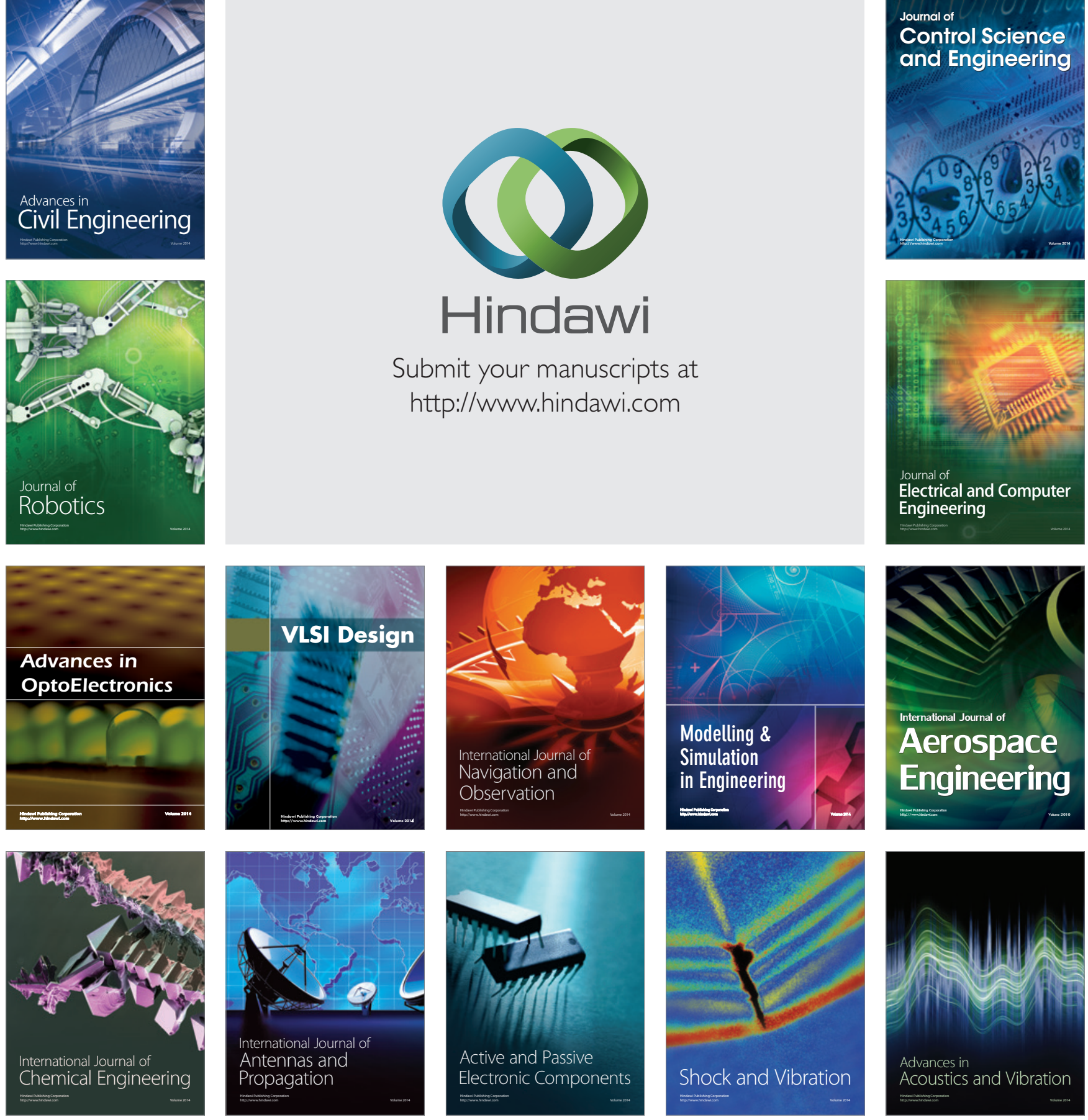